\title{
DER DEUTSCH-RUSSISCHE UNTERNEHMER ANDREAS KNAUF IM URAL
}

\section{Der Aufstieg}

Hans Peter Andreas Knauf, known in Russia as Andrey Andreevich Knauf (1765 - 1835), is an uncommon figure in Russian history. His life leaves many questions that are still to be answered. He was, however, an outstanding personality that played a significant role in the development of the mining and iron foundry industries in the Urals. Born in Kiel, the capital of the Duchy of Holstein, Andrey Knauf, a son of a shoemaker who had neither capital nor connections, was a talented autodidact, and a fellow-countryman of Emperor Peter III. He came to Russia at the age of 18 and achieved considerable success in commerce becoming a prominent merchant of the top guild in Moscow and a manufacturer in the Urals. The article focuses on Knauf's activity in the Urals as an owner of mining factories and on their modernization by transferring German technologies, especially in 1801-1811. His experience is interesting in the context of serfdom and the lack of trained workers in the system of possessional manufactures. However, to study the problem in question the author considers it reasonable not to confine to the meta-concepts of autocracy and serfdom, which are perceived as interpretative patterns for the period in question, but to complete those meta-concepts with micro-historical sketches focusing on "particular" facts and the role of an individual in history.

Keywords: late $18^{\text {th }}$ - early $19^{\text {th }}$ century; P. M. Gusyatnikov; A. Knauf; I. M. Luginin; N. E. Myasoedov; A.B. Kurakin; M. F. Soymonov; I. F. Völkner; A. N. Golitsyn; L. N. Gagarin; G. A. Stroganov; S. P. Yaguzhinskiy; I. P. Osokin; S. A. Fomintsov; industrialization; mining industry; metal-working industry; Urals; modernization; transfer of technologies; transfer of knowledge.

Hans Peter Andreas Knauff, in Russland Andrej Andreevič Knauf(17651835), ist ein ungewöhnliches Phänomen in der russischen Geschichte, das bis heute viele Fragen aufgibt, auf die es nur annähernd zufriedenstellende Antworten geben kann. Eines kann jedoch als sicher gelten - Andrej Knauf ist ein ungewöhnlicher Unternehmer in der Geschichte des Bergbaus und der metallverarbeitenden Industrie im Ural und darf eine herausragende Persönlichkeit genannt werden.

In Kiel geboren, wurde er zum russischen Untertan, Moskauer Kaufmann 1. Gilde und Inhaber und Pächter von zahlreichen Bergwerken, 
Eisenhütten und Metallwarenfabriken. Im Kontext seiner unternehmerischen Tätigkeit ist es interessant, Aspekte einer Modernisierung der Metallindustrie in Russland zu untersuchen. In die Zeit, als Andrej Knauf von 1797 bis 1818 Berg- und Metallwerke am Ural leitete, insbesondere in der Kernzeit von 1801 bis 1811, gehören wichtige Impulse für eine Modernisierung, die die Entwicklung der metallverarbeitenden Industrie positiv beeinflusste.

Seine Erfahrungen geben eine indirekte Antwort auf die Frage, die auch heute viele bewegt: Wie ist es eine Modernisierung zu bewältigen, wenn mikro- und makroökonomische Rahmenbedingungen einer Leibeigenschaft und staatlicher ineffektiver Verwaltung die Geschickte der Wirtschaft in vielem bestimmen? Diese Frage hat an Aktualität in keiner Weise verloren. Raison d'etat und private unternehmerische Initiative, die oft in einen Interessenkonflikt gerieten, geben im Vergleich zu heute verblüffend ähnliche Sachverhalte aus dem Ende des 18. Jahrhunderts wieder. Die brisante Frage in Russland, wie viel Staat und wie viel privatwirtschaftliche Initiative überhaupt sein soll, bleibt nach wie vor hoch aktuell.

Der Autor dieser Fallstudie geht davon aus, dass weder „Autoritarismus" noch "Leibeigenschaft" ausschließlich am Erfolg oder Misserfolg eines Unternehmens „schuld“ waren, sondern vielmehr eine komplizierte Kombination vieler Faktoren.

\section{Die Kaufmannslehre in St. Petersburg und die kaufmännische Praxis in Moskau}

In der Sekundärliteratur sind vor allem zwei Artikel zu erwähnen, deren Autoren wichtige Forschungsarbeit geleistet haben: Erik Amburger und Ewgenij G. Nekljudow. [Amburger, 1982, S. 122-130; Неклюдов, c. 83-101] Ihre Artikel bilden eine wichtige Grundlage zur Erforschung dieses Themas.

„Der 1765 in Kiel geborene Schuhmachersohn Hans Peter Andreas Knauff begleitete seinen Vetter Hans Friedrich Knauff, als dieser 1784 als Silberschmiedgeselle nach St. Petersburg reiste, wo man Verwandte besaß und wo Hans Friedrich es bald zum Meister brachte, während der jüngere Andreas in die Kaufmannsgilde ging. " [Amburger, 1982, S. 122] Es kann als sicher gelten, dass Andreas Knauf ein Jahr früher schon Kiel verließ. Dies bezeugt sein Pass, der am Samstag, den 19. Juli 1783, vom Oberpräsidenten der Stadt Kiel Hans Casper Reichsgraf von Bothmer ausgestellt wurde, damit Knauf nach St. Petersburg reisen konnte [ЦИАМ, ф. 32, оп. 4, д. 2758, 1. 3]. ${ }^{1}$ Knapp eine Woche später, am Freitag den 25. Juli, ging der junge Mann in St. Petersburg an Land [ЦИАМ, ф. 32, оп. 4, д. 2758, л. 3].

In vier Jahren absolvierte Andrej Knauf seine kaufmännische Lehre in der neuen russischen Hauptstadt und siedelte 1788 mit 23 Jahren nach

\footnotetext{
${ }^{1}$ Ca. eine Woche dauerte eine Reise mit dem Schiff von Kiel nach St. Petersburg.
} 
Moskau über, wo er eine Anstellung im Kontor von John Tamesz \& Co., einer alten Export- und Importfirma holländischen Ursprungs, fand [Amburger, 1979, S. $161 \mathrm{f}$ ]. Hier in Moskau war er so erfolgreich, dass er in sieben Jahren eine gute Position im Handelsgeschäft erreichte und 1795 mit seinem englischen Partner William Doughty die Großhandelsgesellschaft Doughty, Knauff \& Co. gründen konnte. Dafür traten beide in die erste kaufmännische Gilde ein: A. Knauf am 19. Dezember im Basmanner Bezirk, in dem die Großkaufleute Mitglied waren [Микитюк, с. 71]. Dazu mussten beide Kaufleute die russische Staatsangehörigkeit erwerben, andernfalls hätte man ihnen nur eine Mitgliedschaft auf Zeit gewährt. Um jedoch uneingeschränkt Geschäfte tätigen zu können, bedurfte es der ständigen Mitgliedschaft. Doughty nannte sich von nun an Vasilij Vasiljevich. Knauf hieß in der Meldebescheinigung aus dem Jahr 1795 immer noch „Petr Andreev syn Knauf“, wobei in seinem Pass unter dem Vermerk vom 2. September 1793 auf Russisch Andrej Andreevich beglaubigt wird [ЦИАМ, ф. 32, оп. 4, д. 2758, л. 12].

Beim Erwerb der russischen Staatsangehörigkeit gab es zwei wesentliche Momente. Aufgrund des Hoheitlichen Erlasses vom 31. August 1795 schrieb der Moskauer Magistrat Andrej Knauf am 4. September als zeitweiliges Mitglied in die Kaufmannsgilde ein [Ibid., л. 8]. Kurz danach reichte er einen Brief an die Kaiserin mit der Bitte ein, ihm eine "ewige russische Staatsangehörigkeit" zu verleihen und zu erlauben, sich als ständiges Mitglied in die 1. kaufmännische Gilde einzuschreiben [Ibid., л. 11]. Schließlich wurde er am 17. Dezember des gleichen Jahres vom Pastor der evangelisch-lutherischen Peter-Paul-Kirche im Basmannaja Bezirk vereidigt. Seinen sozialen Aufstieg setzte Knauf 1801 fort, als er zum "namhaften Bürger" (imenityj grazhdanin) der Stadt Moskau ernannt wurde [ПС3-1, т. 26, c. 326, zitiert nach: Amburger, 1982, S. 53]. ${ }^{2}$ Damit gehörte er der obersten Klasse der nichtadeligen städtischen Gesellschaft.

Peter Andreas Knauf ließ also seinen Namen erst am 31.12.1801 offiziell ändern, wobei er auch vorher schon, z. B. im Jahr 1788 bei den russischen Behörden, Andrej Andreevich genannt wurde und seine Gesuche auch mit diesem Namen unterschrieb. [ЦИАМ, ф. 32, оп. 4, д. 2758, л. 3, 15]. ${ }^{3}$ Das war eine verbreitete Praxis unter den Ausländern, die sich zur Erleichterung des Umgangs mit den russischen Kollegen fast immer einen russischen Namen zulegten. Der Eintritt in die 1. Gilde ermöglichte Knauf und seinem Partner, nicht nur intensive Handelsgeschäfte zu tätigen, sondern auch ein industrielles Gewerbe zu betreiben. Damals wohnte Knauf im Hause des General-Leutnants S. A. Fomintsov nicht weit vom Borovitskij Kloster [Микитюк, с. 71].

Die ersten Erfahrungen konnten die beiden Partner 1796 machen, als ihnen am 25. Februar die Verwaltung des Preobrazhensker Werks des Mos-

\footnotetext{
${ }^{2}$ Identisch mit dem Stand der Ehrenbürger, der im Jahr 1832 eingeführt wurde.

${ }^{3}$ Auf der Rückseite sind auch Vermerke aus den Jahren 1783, 1788 und 1793 zu finden.
} 
kauer Kaufmanns Petr Michajlovich Gusjatnikov aufgetragen wurde. Dies kam zustande, als die Bankkaufleute John Hembry und Karl Hasselgreen und der englische Kaufmann Schneider vermutlich als Gläubiger von Gusjatnikov teilweise Eigentumsrechte an dessen Besitztum bekamen [РГИА, ф. 37, оп. 3, д. 187, л. 1-64; vgl.: РГИА, ф. 1374, оп. 3, д. 2649].

Andrej Knauf witterte große Geschäfte. Vielleicht schwebte ihm ein ähnliches Schicksal wie das von Nikita Demidov und seinem Sohn Akinfij vor, die ein Industrieimperium am Ural aufbauten und es zu großem Reichtum brachten. Bis etwa 1807 gelang dies auch Knauf gut, dann ging es allerdings bergab. Bis dahin waren es jedoch etliche Jahre unglaublicher Expansion: Knauf und seine Partner kauften, wo es möglich war, die Metallhütten im Ural auf.

\section{Der Aufstieg}

Der erste große Erwerb durch Doughty und Knauf fand im Jahr 1797 statt. Der russische Kaufmann Ivan M. Luginin war in finanzielle Nöte geraten und hatte sich immer mehr verschuldet. Vermutlich verfiel er der Spiel- oder Trunksucht. [РГИА, ф. 1374, оп. 2, д. 1779, л. 6; РГИА, ф. 1374, оп. 2, д. 1781 $]^{4}$. Ihm blieb nichts anderes übrig, als seine Zlatouster Werke im Südural 1797 zum Verkauf anzubieten [Ibid., д. 1799, л. 9, $48 f] .{ }^{5}$ Der Preis betrug 1.800.000 Rubel [Ibid., л. 2] ${ }^{6}$. Schließlich wurde am 26.11.1797 ein Kaufvertrag zwischen Luginin und Knauf in Anwesenheit vom Luginins Vormund N. E. Mjasoedov unterzeichnet, nach dem Knauf fünf Werke sowie dazugehörige Possessionsbauern und Ländereien bekam [РГИА, ф. 1374, оп. 2, д. 1779, л. 48]. ${ }^{7}$ Dieser Vertrag wurde jedoch nicht von höchster Stelle bestätigt, sondern rückgängig gemacht.

Major Ahmatov, ehemals Luginins Leibeigener und Leiter seiner Werke, und der andere Werksleiter Major Hrushchev erfuhren vom Vertrag und meldeten ihre Ansprüche beim Bergkollegium, Senat und dem Zaren selbst. Dabei zweifelte man in der Regierung an der Eignung dieser Bewerber. Es war bekannt, dass sich die beiden eines unrechtmäßigen Erwerbs erfreuten. Ahmatov „bereicherte [...] sich auf Kosten von Luginin und hat jetzt ein beträchtliches Kapital. Er versucht eine fürsorgliche Rolle zu spielen, um an den Besitz der Werke heranzukommen, in Wahrheit aber strebt er einen vollkommenen Ruin von Luginin an, während er auch in

\footnotetext{
${ }^{4}$ Siehe auch den Brief von Nikolaj Mjasoedov vom 25. August 1799. Luginins Ehefrau lebte von ihrem Mann getrennt und mittellos in St. Petersburg.

${ }^{5}$ Am 25.08.1799 schrieb Nikolaj Mjasoedov in einer Erklärung: „Als ich jedoch meine Vormundschaft aufnahm, verließ Luginin sündhaftes Leben und zeigt bereits seit zwei Jahren gute Führung, wie es sich bei einem edlen Mann gehört."

${ }^{6}$ Mjasoedov erwähnt, dass der anfängliche Preis bei 1.370.000 Rubel lag und es ihm gelang, Knauf dahin zu bringen, den höheren Preis zu zahlen und noch zusätzliche 25.000 Rubel für dringende Maßnahmen zwecks Aufrechterhaltung des Betriebs „dieser verfallenden Werke“.

${ }^{7}$ Die Possessionsbauern durften nicht getrennt von den Werken verkauft werden, weil sie ihre Arbeit dort entrichten sollten. Das konnten sowohl die Leibeigenen als auch die staatlichen Bauern sein, deren elende Lage sich jedoch kaum voneinander unterschied.
} 
den Besitz von Luginins Wechseln für 112.000 Rubel herangekommen ist. Er hat ihn im höchsten Maße bestohlen und trieb ihn in die Pleite" [Ibid., л. 8]. Unter dem Vorwand der Fürsorge kaufte Ahmatov von Luginin die Ein-Rubel-Wechsel für 20 Kopeken ab, verlangte jedoch dafür die ganze Summe von 112.000 Rubeln. Auch Major Hrushchev kam auf die gleiche Weise in den Besitz eines Wechsel von 22.000 Rubeln. In diesem Fall bekam Luginin nur 2.000 Rubel [Ibid.].

Das langwierige Erwerbsprozedere der Werke von Luginin nennt Mikitjuk zurecht als widersprüchlich und geheimnisvoll [Микитюк, с. 72]. Dabei lässt sich nach den letzten Erkenntnissen sagen, dass es hier fünf Interessenparteien gab, die eine wesentliche Rolle spielten: der Verkäufer Nikolaj Luginin, die Käufer Andrej Knauf und Vasilij Doughty, sowie die russische Regierung, außerdem die russischen Mitbewerber Majoren Ahmatov und Chrushchow. Die vielen Interessenten und der launige Kaiser Paul I. konnten für viele überraschende Wendungen sorgen.

Die Geschichte des Erwerbs durch Andrej Knauf lässt sich mit Hilfe eines Berichts aus der Kanzlei des General-Prokurors im regierenden Senat Alexej Kurakin rekonstruieren [РГИА, ф. 1374, оп. 3, д. 2500, л. 2-4]. Darin wird es erklärt, dass Knauf in den Ruin getrieben werde, weil die bei Luginin erworbenen Werke von der Krone wieder zurück gekauft wurden, nachdem Knauf bereits einen Kaufvertrag abgeschlossen hatte. Darin verpflichtete sich Knauf, die Werke für 1.800.000 Rubel zu kaufen, sofort deren Leitung zu übernehmen und darüber hinaus mit 400.000 Rubel in Vorlage zu treten, um die Luginins Gläubiger zu entschädigen und die Aufrechterhaltung des Betriebs der Werke zu garantieren [Ibid., л. 2]. Vor allem brauchte man Geld für die Verpflegung und die Löhne der Werksarbeiter.

Dafür lieh sich Knauf 1.000.000 Rubel im Ausland. Der regierende Senat bestätigte zwar den Kaufvertrag und legte ihn dem Zaren zur Unterzeichnung vor, was General-Prokuror Kurakin, der gleichzeitig Direktor der Reichsassignatenbank war, nicht hinderte, einzugreifen und zu erklären, Knauf sei ein Ausländer und kein russischer Untertan und habe deshalb kein Recht, solche Kaufverträge abzuschließen. Das entsprach jedoch nicht der Wahrheit, denn Knauf war seit 1795 russischer Untertan.

Desweiteren trug die Entdeckung des Goldvorkommens am Werk in Zlatoust dazu bei, dass die Regierung nach Abschluss des Kaufvertrags durch Knauf das Ganze für ungültig erklärte. So blieb die Goldgewinnung bis 1812 Staatsmonopol.

Danach wurde Luginin von höchster Stelle befohlen, alles daran zu setzen, die Werke zu behalten und den Betrieb zu gewährleisten. Dafür stellte ihm die Reichsleihbank ein Darlehen zur Verfügung. Sollte Luginin scheitern, würden die Werke von der Krone unter den gleichen Bedingungen wie bei Knauf gekauft werden [РГИА, ф. 1374, оп. 3, д. 2500].

Dies bedeutete für Knauf einen Verlust von 300.000 Rubel, die Zinszahlungen und die Rückgabe des Kredits eingerechnet. Als Knauf seine russische Staatsangehörigkeit bewies, bekam er von der Krone 100.000 Rubel zurück. 
Widersprüchlich war das Verhalten seitens der russischen Regierung, als sie die Werke für sich beanspruchte, sie dann aber 1797 sofort zum Verkauf anbot, um die Schulden an die Gläubiger zurück zu zahlen [РГИА, ф. 1374, оп. 2, д. 1779, л. 10, 19] ${ }^{8}$. Anderthalb Jahre passierte nichts [л. 8]. ${ }^{9}$ In dieser Zeit wurde Knauf, der sich im rechtmäßigen Besitz der Werke sah, mit weiteren Ausgaben belastet. Das waren unter anderem Ausgaben für die Arbeiter und Bauern. Mjasoedov berichtet über die Zeit, als er den Kaufvertrag mit Knauf abschloss, dass die Werke nur noch für einen Monat Geld hatten, um die Arbeiter mit den nötigen Lebensmitteln zu versorgen. Danach sollten sie stillgelegt und dem Verfall preisgegeben werden. Knauf rettete diese Werke, indem er ein schwieriges Geschäft mit dem Staat einging und sich sehr großzügig zeigte.

Insgesamt lässt sich sagen, dass das Intermezzo mit Knauf und sein energisches Eingreifen um den Erhalt der Lugininschen Werke positive Ergebnisse zeigten. Die Werke waren vor dem Konkurs gerettet, die Produktionsraten stiegen kontinuierlich: In der zweiten Hälfte des Jahres 1797 wurden 75251 Pud Eisen produziert, im Jahr 1798 - 291799 Pud, in der ersten Jahreshälfte 1799 - 287.924 Pud. Jedes Jahr wurde die Produktion verdoppelt, so dass 1799 das eingeplante Volumen mit zusätzlichen 3111 Pud übererfüllt wurde. Die Produktion beinhaltete unterschiedliche Profile, u.a. Eisenblätter und -balken. [Ibid., оп. 3, д. 2500 , л. $36 \mathrm{f}$ ].

Nach allen Gesuchen und Bitten wurde dem Direktor des Bergkollegiums M. F. Sojmonov ${ }^{10}$ auferlegt, die Angelegenheit um die Lugininschen Werke endlich zu regeln. Erschwerend kam hinzu, dass Kurakin und Sojmonov mit Gesuchen von Ahmatov und Hrushchev konfrontiert wurden. Letztere waren der gleichen Meinung, dass es sich nicht gehöre, die Werke in private Hände zu geben. [РГИА, ф. 1374, оп. 3, д. 2500, л. 3]. Von Bedeutung war auch, dass Fürst Kurakin dem Bergrat angehörte. [Тулисов, 1999, с. 33-36; ГАСО, ф. 24, оп. 1, д. 2982, л. 73]. Die Bittschrift von Luginin, seinem Kaufvertrag mit Knauf stattzugeben, sowie die Bittschriften von Knauf, Ahmatov und Hrushchev wurden von der höchsten Stelle abgelehnt. [Ibid., оп. 2, д. 1779, л. 8].

Kurakin und Sojmonov sorgten dafür, dass die Werke in kürzester Zeit von der Reichsassignatenbank, die gegenüber der Bank-Brücke mit den Löwen-Greifen stand, zurück gekauft wurden. Wiederum trug die Reichsassignatenbank die Verwaltung der Werke dem Bergkollegium auf [Ibid., on. 3, g. 2500, л. 4]. Hiermit übernahm der Staat in Person von Kurakin nahezu die gesamte Verwaltung der Werke und brachte sie gänzlich in den eigenen Machtbereich.

\footnotetext{
8 „...Weil aber Luginin durch seine Schwäche in kürzester Zeit $<\ldots>$ große Schulden gemacht hatte“. Die Schulden betrugen nahezu zwei Millionen Rubel.

${ }^{9}$ Aus dem Brief von Nikolaj Mjasoedov vom 25.08.1799.

${ }^{10}$ Präsidenten des Bergkollegiums waren: M. F. Sojmonov (1771-81), I. I. Rjazanov (1781-1784), A. A. Nartov (1796-1798), A. V. Aljabjev (1798-1802), A. I. Korsakov (18021806). In: Geologičeskaja ènciklopedija, Artikel von V. A. Bojarskij. URL: http://dic.academic.ru/dic.nsf/enc_geolog/464/\%D0\%91\%D0\%B5\%D1\%80\%D0\%B3.
} 
Damit war die Geschichte noch nicht zu Ende, denn der Verkauf des Eisens seitens der Reichsassignatenbank wollte nicht vonstatten gehen: „Sehr langsamer Verkauf des Eisens in kleinen Mengen, das sich in der Handelsbörse angehäuft hat, führen die Unbedarftheit der Staatskasse in solchen Handelsangelegenheiten deutlich vor Augen. Es gehört sich nicht, die Staatskasse nach den Bräuchen der Handelsleute zu agieren." [РГИА, ф. 1374, оп. 3, д. 2500, л. 4]. Das Fazit lautete: „Eine Analyse der letzten zehn Jahre zeigt, dass die Bergwerke und Eisenhütten, die in privater Hand liegen, eine höhere Effektivität aufweisen." [Ibid.].

Das Bergkollegium nahm plötzlich den Standpunkt von Andrej Knauf ein und pflichtete ihm bei, dass „die Werke in der Staatshand nicht gewinnbringend betrieben werden können und die einzige Lösung wäre, sie Knauf zurück zu geben." [Ibid.]. Die Regierung könnte sich in diesem Fall als Gewinner betrachten, da Knauf sich verpflichtete, ihr das Geld, das sie für den Kauf der Werke aufgewendet hatte, zurückzuerstatten. Es bedurfte nur der Genehmigung des Kaisers.

Knauf kämpfte weiter und erreichte, dass die Werke ihm nach dem kaiserlichen Erlass vom 4. Mai 1798 zur Leitung und zur Pacht übergeben wurden. Chrushchev wollte im Auftrag der Regierung die Werke leiten und bot 1000 Leibeigene als Pfand. Ahmatov bot 2000 Leibeigene und wollte als selbständiger Unternehmer die Leitung übernehmen. Knauf bot dazu noch seine Firma als Pfand an. Das genügte, um das Bergkollegium und den Senat zu überzeugen [Ibid., л. 19], allerdings unter der Bedingung, dass Knauf das fünfte Werk in Zlatoust, die Kupferhütte mit Ländereien und Arbeitern, dem Staat abtrat [РГИА, ф. 1374, оп. 3, д. 2500]. ${ }^{11}$ Auch diesmal wollten sich Ahmatov und Hrushchev nicht geschlagen geben und ersuchten den Zaren, ihnen jeweils eines der Werke als Pächter zu überlassen. Sie bezweckten damit, „das Ganze zu verwirren und zu verhindern." [Ibid., оп. 2, д. 1779, л. 8]. Dies hatte zur Folge, dass die Zlatoust Werke am 20.05.1799 wieder von der staatlichen Reichsassignatenbank gekauft wurden [Ibid., л. 54]. Ein anderer Deutsche, I. F. Völkner, wurde von der Regierung zum Leiter der Werke ernannt. Er blieb im Amt von Juli 1799 bis April 1802, als die Werke bereits wieder Knauf gehörten. [Новиков; Неклюдов, с. 83-101; vgl.: ГАСО, ф. 24, оп. 3, д. 72, л. 2, 14]. Die seit 1797 kontinuierlich steigenden Produktionszahlen wuchsen auch unter dem neuen Leiter weiter. Sehr wahrscheinlich arbeitete Völkner in enger Verbindung mit Knauf. Zum wiederholten Male begannen die Staatsmühlen zu mahlen.

In der Zwischenzeit ging Völkner die Leitung der Werke energisch an. Wie es sich zeigte, verlief auch hier die Konfliktlinie zwischen dem neuen Hauptleiter und den einzelnen Leitern der Werke wie E. F. Ahmatov. Dieser beschuldigte Völkner nämlich eines Amtsmissbrauchs, einer bewussten Reduzierung der Produktion und einer moralischen Verderbt-

\footnotetext{
${ }^{11}$ Später hat es sich erwiesen, dass dort kein nennenswertes Goldvorkommen vorhanden war, die Kupferhütte blieb jedoch in staatlicher Hand, was für Knauf einen großen Verlust bedeutete.
} 
heit, was nach einer Überprüfung nicht bestätigt wurde [Тулисов, 1999, c. 273-274]. In der Tat führte Völkner nach seiner Amtseinführung am 25. Mai 1799 ein strenges Regiment. Als er am 10. Juli vor Ort eingetroffen war, stellte er Inventarlisten zusammen und überprüfte die Werksunterlagen und Arbeiterzahlen. Ahmatov, dem Verwalter des Werks Artinsk, wurde wegen seiner Willkür und des eigenwilligen Gebrauchs des staatlichen Eigentums gekündigt. Er kam vor Gericht. Zvezdin, Verwalter des Werks Miass, und sein Buchhalter wurden für die Unterschlagung staatlicher Gelder bestraft [Новиков]. Die wieder hergestellte Ordnung in den Werken zeigte sich in den Produktionszahlen. Es wurden im Zlatouster Werk jährlich 280000 bis 416000 Pud Roheisen produziert. Völkner wie auch später Knauf beschwerte sich wegen des Mangels an Fachleuten, konnte jedoch wenig ausrichten. Einmal begegneten sie sich noch im Jahr 1812 auf dem Jahrmarkt in Makarjev bei Nizhnij Novgorod. Der ältere Sohn von Völkner, Fjodor, war 1856-1863 Hauptleiter der Bergwerke im Ural und hatte unmittelbar mit den Knauf-Werken zu tun [Ibid.].

Die Schwierigkeiten beim Erwerb der Lugininschen Werke durch Knauf hatten unter anderem mit der Umstrukturierung der staatlichen Bergverwaltung zu tun. [Тулисов, 1999, с. 33-36; ГАСО, ф. 24, оп. 1, д. 2629, л. 166]. Aufgrund der Erlasse vom 4.1.1797 und 3.5.1797 bekamen die neuorganisierte Kanzlei der Hauptverwaltung der Bergwerke Ural und ihr Hauptleiter mehr Befugnisse. Die Regierung behielt den Ural mit seiner strategischen Bedeutung besonders für die Kriegsindustrie, immer im Blick. Um diese Zeit wurden dort 81\% Eisen und 95\% Kupfer hergestellt [Ibid.]. Das war die einzige Region in Russland, in der Gold gefördert wurde. Verständlich, dass diese Region große Begehrlichkeiten von allen Seiten weckte. So gewannen in der Regierung abwechselnd prostaatliche und privatwirtschaftliche Interessen die Oberhand, nicht zuletzt je nachdem, wo die Staatsdiener mehr Vorteile für sich und für die Staatskasse erhofften.

Es folgen Bittschriften von Knauf an höhere Stellen über die Rückgabe der Werke. Eine davon ist mit 25.05.1800 datiert. Knauf erwähnt darin, dass er außer 300.000 Rubel noch Verluste in den Wechseln durch die Pleiten in Hamburg und London zu verzeichnen hätte. Seinerseits erklärte er sich bereit, nicht nur auf die Rückerstattung seiner Verluste zu verzichten, sondern auch alle Vorräte an Eisen, das unter seiner Leitung hergestellt worden war, an den russischen Staat abzutreten. Darüber hinaus erklärte er sich bereit, zusätzlich zur fälligen Steuer jährlich 100.000 Rubel und weitere $10 \%$ an die Staatskasse zu zahlen. Ebenso verpflichtete er sich, das Eisen, das bei der Reichsassignatenbank deponiert wurde, zum höheren als auf der Börse gehandelten Marktpreis zu kaufen [РГИА, ф. 1374, оп. 3, д. 2500, л. 8, 22, 42, 63]..$^{12}$ Dass Knauf bereit war, solch hohe Auflagen auf sich

\footnotetext{
${ }^{12}$ Knauf wurde verpflichtet, 287.517 Pud Eisen für 2 Rubel pro Pud für 575.034 Rubel insgesamt zu kaufen. Im-Endeffekt zahlte er dann noch mehr - 706.868 Rubel. In seinem Gesuch aus dem Jahr 1812 nennt er gar 350.000 Pud.
} 
zu nehmen, zeigt, dass er um jeden Preis sein Ziel erreichen wollte. Er hatte dazu auch alle Möglichkeiten: Hinter diesem Projekt standen außer ihm einflussreiche ausländische Kapitalgeber, die offensichtlich daran interessiert waren, beträchtliche Mittel in die russischen Berg- und Hüttenwerke zu investieren.

Aber es gab auch schmerzliche Verluste: Im Jahr 1800 fuhr Knaufs Partner Doughty zurück nach England und die Firma hieß von nun an Knauf \& Co. Knauf erwies sich als hartnäckig, und nach fast anderthalb Jahren wurde der endgültige Erlass vom 30.09.1800 veröffentlicht, Kraft dessen ihm die Werke übergeben werden sollten [РГИА, ф. 1374, оп. 3, д. 2500, л. 27, 59-80; ГАСО, ф. 24, оп. 3, д. 72, л. 23; ПС3-1, т. 26, № 19583 , с. $326 f]^{13}$. Dadurch bekam Knauf den ganzen Zlatouster Bergbaubezirk unter seine Verwaltung [РГАДА, ф. 271, оп. 1, д. 2978, л. 11-18; РГИА, ф. 560, оп. 3, д. 96, л. 2] ${ }^{14}$ Außer der Berg- und Metallwerke trug er die Verantwortung für 5245 Arbeiter und Possessionsbauern [РГИА, ф. 1374, оп. 2, д. 1779, л. 15] ${ }^{15}$ sowie ihre Familienmitglieder, insgesamt ca. 12000 „Seelen“, bekam Ländereien, Wälder und Bodenschätze wie Eisenerz [Микитюк, с. 72; РГИА, ф. 1374, оп. 2, д. 1779, л. 7]. Außerdem zählte er seit dem 31. Dezember $1801 \mathrm{zu}$ den Ehrenbürgern der Stadt Moskau [ЦИАМ, ф. 2, оп. 2, д. 70, л. 89 f]. Rechtlich gesehen war er ein erblicher Pächter, zuerst auf zehn und dann auf 25 Jahre, der sich verpflichtete, alle Schulden innerhalb von zehn Jahren abzuzahlen. Seine ehrgeizigen Pläne schienen in Erfüllung zu gehen. Die Werke wurden auf 25 Jahre an die Reichsassignatenbank verpfändet, bis Knauf alle Schulden, die Luginin gemacht hatte - und die verliefen auf ca. 2.000.000 Rubel, Zinsen inklusive - an die Gläubiger zurück gezahlt hatte [РГИА, ф. 1374, оп. 3, д. 2500, л. 19]. Das fünfte Werk, in dem Goldvorkommnisse gefunden wurden, trat Knauf an die Krone ab. Sie machte damit ein gutes Geschäft, da Knauf bereit war, auf die Rückerstattung der Schulden durch Prozesskosten in Höhe von 400.000 Rubel, zu verzichten und jährlich an Luginin eine Pension in Höhe von 20.000 Rubeln zu zahlen. [Ibid., оп. 2, д. 1779, л. 1, 4ff].

$\mathrm{Zu}$ dieser Zeit verfügte Knauf bereits über Zugang zu den einflussreichen russischen Adeligen. Sein Verhalten lässt Rückschlüsse auf seinen Charakter zu. Dass er nicht nur sehr zielstrebig und energisch, sondern auch fähig war, Sympathien zu gewinnen, bezeugte sein immer größer werdender Partner- und Bekanntenkreis. Er gestaltete raffinierte Kombinationen, genoss großes Vertrauen und konnte überzeugen, galt gar als ein Wirtschaftsgenie. Hinter ihm standen die Bankiers und Großkaufleute Doughty, Schneiders und Hasselgreen. Ihn unterstützten einflussreiche russische Kaufleute und Adelige. Dazu gehörten Fürst A. N. Golitsyn,

\footnotetext{
${ }^{13}$ Knauf wurde der Vorzug mit der Begründung gewährt, weil dass er „Kenntnisse in der Bergwissenschaft besitzt“.

${ }_{14}$ Werke: Artinskij, Zlatoustovskij, Kusinskij und Satkinskij; vgl.: [Amburger, Knauff, S. 122f.]; Неклюдов, с. 83-101].

${ }^{15}$ Stand am 29.06.1798.
} 
Fürstin L. N. Gagarin, Baron G. A. Stroganov, Graf S. P. Jaguzhinskij, die Industriellen I. P. Osokin und P. M. Gusjatnikov. Er war so ehrgeizig, dass er gegen die russische Regierung einen Prozess anstrengte und ihn auch gewann. Ein Großunternehmer sollte sich jedoch früher oder später mit der Krone arrangieren.

Als Pfand für die Werke durfte er laut Kaufvertrag 1000 leibeigene Bauern „hinterlegen“. Da er aber keine hatte, erklärte sich Fürst A. N. Golitsyn bereit, ihm seinen Gutsbesitz in Kaluga zu verkaufen [РГАДА, ф. 271, оп. 1, д. 2989]. Bis der Kaufvertrag in Kraft trat, durfte Knauf wiederum eine Vollmacht von der Fürstin L. N. Gagarin vorlegen, die 1000 Leibeigene aus ihren Gütern in drei Gouvernements zur Verfügung stellte. [Неклюдов, с. 83-101].

Drei Jahre später, am 16. September 1804, überließ Baron G. A. Stroganov Knauf drei Eisenhütten im Ural, auf denen bereits 470.000 Rubel Schulden lasteten. [Amburger, 1982, S. 126]. Dafür verpflichtete Knauf Stroganov zur Zahlung einer einmaligen Summe von rund 500.000 Rubel. Stroganov schätzte Knauf als einen fähigen Manager, den er „von der besten Seite durch seine aktuelle Leitung der (Zlatouster, A. K.) Werke“ kannte. Der Baron erlaubte Knauf, „Werke zu besitzen und Arbeiter zu leiten, als ob das seine eigenen wären, in der festen Hoffnung, dass er dadurch sein ihm zustehendes Gewinn bekommt und dass er nicht ihn, Stroganov, und seine Leute vergesse." [Неклюдов, с. 83-101].

Andrej Andreevič war gut im Geschäft. Schon am 4. April des gleichen Jahres gelang es ihm, einen Kaufvertrag mit dem hoch verschuldeten Industriellen und Moskauer Kaufmann I. P. Osokin abzuschließen und so in den Besitz von weiteren drei Eisenwerken und drei Kupferhütten zu kommen. Dies kostete Knauf 1.315.000 Rubel. [Неклюдов, с. 83-101; Amburger, 1982, S. 126] ${ }^{16}$.

Anfang 1805 leitete Andrej Knauf 14 Eisenwerke und Kupferhütten, die Hälfte davon in seinem Besitz, die andere Hälfte gepachtet. Dank dieses Erwerbs zählte die Firma Knauf \& Co. zu den dynamischsten in der Branche. Dies kostete Knauf oder besser gesagt seinen Gläubigern viele Millionen Rubel an Investitionen. Die Tatsache, dass es Knauf gelang, über Jahre hinweg sein Unternehmen aufrecht zu erhalten und die Produktion sogar zu steigern, verdient alle Achtung und ist einzig und allein Knaufs unternehmerischem Talent zuzuschreiben. Der Kapitalmangel, unter dem sein Unternehmen permanent litt, wurde ihm schließlich zum Verhängnis [Микитюк, с. 73].

\section{Zum Verhältnis von Staat und Privatunternehmen in der Hüttenindustrie}

Das Geschehen mit und um Andrej Knauf kann nur im Kontext der Entwicklung im 18. Jahrhundert verstanden werden. Von Anfang an ging

\footnotetext{
${ }^{16}$ Beim aktuellen Wechselkurs von 249 Rubeln für einen Silberrubel oder 90 Rubeln für eine Banknote durften das 327.435.000 oder 118.350.000 Rubel sein.
} 
die Gesamtentwicklung von Hüttenwerken und metallverarbeitender Industrie Hand in Hand mit staatlicher Initiative und Unterstützung. Peter der Große legte die Grundlagen dieser Industrie, wobei sie schon im 17. Jahrhundert mithilfe ausländischer Unternehmer in Tula und Karelien entwickelt wurde. Eine Zäsur markierte die Gründung des Bergkollegiums mit dem Erlass vom 10.12.1719, das die Bergindustrie einschließlich der Hüttenwerke leiten sollte. [ПС3-1, т. 5, №. 3464, c. 760ff]. Für Unternehmer und Handwerker bedeutete dies Vergünstigungen und Steuererleichterung. Dafür behielt sich der Staat das Recht vor, die Betriebe zu kontrollieren und alle Produkte der Werke zu kaufen. Nur dann, wenn die Staatskasse dazu nicht imstande war, durfte an Kaufleute weiterverkauft werden. Alle Werke wurden aus der Jurisdiktion der Gouverneure herausgenommen und unterstanden unmittelbar dem Bergkollegium. Weil eine Industrie dieser Art früher nur in Ansätzen existierte, kann dies als ein wesentlicher Fortschritt bezeichnet werden. Im 18. Jahrhundert entsteht dadurch vor allem am Ural ein großer Industriezweig. In dieses erste Drittel des 18. Jahrhunderts gehören Namen von Industriellen wie Demidov, Stroganov, Vjazemskij, Osokin, Nebogatov, Turchaninov. Mit Stroganov und Osokin schloss Knauf seinerzeit Verträge.

Die Entwicklung dieser Industrie verlief auch unter ständiger, wohlgesagt ungleicher, Konkurrenz zwischen Staat und privatem Unternehmertum. Vieles hing davon ab, welche Sicht der Dinge die Regierung hatte. Die meisten Industriebetriebe, die Peter I. gründen ließ, überlebten ihren Initiator nicht lange. Zu Zeiten Anna Ioannovnas befanden sich die staatlichen Betriebe in einem desolaten Zustand, dagegen wiesen die privaten Betriebe vergleichsweise bessere Zahlen und in den meisten Fällen eine positive Entwicklungsdynamik auf. Der Erlass aus dem Jahr 1739 verfügte deshalb, die staatlichen Betriebe an Privatunternehmer zu übergeben.

Neben staatlichem Besitz erschien 1747 noch eine neue Form vom Besitztum - der Kabinettbesitz. Dieser ging aus einem Präzedenzfall hervor, als die Altai-Werke des verstorbenen Akinfij Demidov unmittelbar in den Besitz der Zarin übergingen. Die jährliche Kupferproduktion in dieser Zeit lag bei ca. 3000 Tonnen. Gleichzeitig bekam der Staat von allen Hütten 10 bis 20\% des produzierten Eisens als Naturalsteuer, 50-70\% der Produktion nahm er den Privatunternehmen zu festen Preisen ab. 1775 wurde der freie Handel mit Kupfer sogar untersagt. Allerdings wies die sogenannte Kabinett-Wirtschaft kaum Wachstum auf, behielt jedoch ihre Position bis in die Mitte des 19. Jahrhunderts.

Die neue Industrialisierungswelle mit den alten Methoden, d.h. kleine Öfen bei großem Arbeits- und Materialverbrauch, kam während der Regierungszeit Katharinas II. auf. In den Jahren 1793-1795 wurden jährlich im Durchschnitt etwa 48.000 Tonnen Eisen produziert [Покровский, c. 99], von denen mehr als die Hälfte nach England exportiert wurde [Fremdling, S. 39]. Die Anzahl der Arbeiter in den Metallwerken am Ural wuchs von $31.000 \mathrm{im}$ Jahr 1719 auf $312.000 \mathrm{im} \mathrm{Jahr} \mathrm{1796.} \mathrm{[Blum,}$ 
p. 311]. Dieses extensive Wachstum erwies sich bald als sehr effektiv: Russland war führend in der Eisenproduktion und deren Export nach Europa. Genau um diese Zeit aber vollzieht sich in Europa ein technologischer Durchbruch durch die kontinuierliche Steigerung der Produktivität von Hochöfen dank der Koksverwendung und effektiver Lüftung mithilfe von Dampfmaschinen als Antrieb für die Gebläse [Бакшаев, c. 6-7, 19]. In Russland blieb man noch Jahrzehnte lang bei der Holzkohle, einem Arbeitsstoff, der sehr zeit- und kostenaufwändig gewonnen wurde und der in Europa sehr teuer und bereits veraltet war. Eine Zäsur bedeutete hier der gelungene Bau eines funktionsfähigen, mit Koks betriebenen Hochofens im Königlichen Hüttenwerk Gleiwitz im November 1796 [Verein, S. 8-9]. Es begann eine Blütezeit der Gusseisenindustrie in Europa [Johannsen, S. 32] - eine verhängnisvolle Entwicklung auch für Andrej Knauf, der, wie wir wissen, intensiv daran gearbeitet hatte, genau in dieser Richtung Verbesserungen in seinen Werken umzusetzen, nämlich bei der Vervollkommnung der Luftzufuhr und der Verwendung von Koks. Bei den veralteten Öfen war es jedoch schwer, wenn nicht unmöglich, Koks zu verwenden.

Zwei Grundfaktoren bestimmten die Entwicklung des Hüttenwesens im Ural: die Leibeigenschaft und der wachsende Export vom Eisen nach Europa, der am Ende des 18. Jahrhunderts seinen Höhepunkt erreichte und von da an wieder zurück ging. Es ist unumstritten, dass die Leibeigenschaft die Grundlage des nationalen Wirtschaftslebens Russlands im 18. Jahrhundert bis 1861 bildete. Das bedeutete vor allem unfreie Arbeit und eine extensive Art des Wirtschaftens. Die Entwicklung in Europa hatte gerade eine gegenläufige Tendenz: Während die Leibeigenschaft in Europa sukzessive abgeschafft wurde, nahm sie in Russland gegen Ende des 18. Jahrhunderts immer mehr zu. Das System war träge, brauchte keine großen Innovationen und war kostengünstig. Die beinahe unendlichen Ressourcen an billiger Arbeit und Naturschätzen erlaubten auch weiterhin eine extensive Entwicklung. Aber schon um diese Zeit versuchte die Regierung das Hüttenwesen zu kontrollieren und zu begrenzen, um den Wald zu schonen. Mit anderen Worten, das Wachstum mit veralteten Technologien stieß schon damals an seine Grenzen. Dennoch konnten die russischen Produzenten die ungeheuren Kosten für die Vorbereitung des Holzes und der Holzkohle und für den Transport des Eisens dank billiger Arbeit immer noch verkraften, was in England schon am Anfang des 18. Jahrhunderts schlecht funktionierte. Der Transport vom Ural bis ins Baltikum nahm ein, im ungünstigen Fall sogar zwei Jahre in Anspruch, wodurch sich das Gusseisen um das Zweieinhalbfache verteuerte [Blum, p. 286]. In Europa war es aber immer noch gewinnbringend zu verkaufen. In Tagiler Hütten beispielsweise wuchs die Anzahl der leibeigenen Arbeiter von 24\% im Jahr 1747 auf 54,3\% im Jahr 1795. Bis 1811 waren alle Arbeiter Leibeigene [Гусякова, с. 30, 37].

Modernisiert wurde überall, die Frage ist, wie viel und wie schnell. Was in Westeuropa einige wenige Jahre dauerte, konnte in Russland Jahrzehnte 
in Anspruch nehmen. Die Überreste der Leibeigenschaft im Hüttenwesen und in der Metallverarbeitung wurden bis zum Anfang des 20. Jahrhunderts noch nicht ganz überwunden. Langfristig gesehen, stand das russische Hüttenwesen auf verlorenem Posten. Während der zweiten Hälfte des 19. Jahrhunderts versiegte der Export aus dem Ural gänzlich [Wallerstein, p. 142; Рожков, с. 41].

Als Knauf nun die Umsetzung seiner Pläne in Angriff nahm, befand sich die russische Schwerindustrie auf ihrem Höhepunkt, und vor ihr lag die lange Stagnationszeit wegen sinkender Eisenexporte nach Europa, was er nicht voraussehen konnte. Erschwerend kam hinzu, dass 1797 die Tendenz zur Zentralisierung der Verwaltung dieses Wirtschaftszweigs am größten war, da die russische Regierung dabei war, die Erträge der Werke zu Gunsten der Staatskasse zu steigern. In Anbetracht der andauernden Kriege in Europa sollte auch die Kriegskasse gefüllt werden.

Mit der Schaffung der Kanzlei der Bergindustrie im Ural kamen drei Goldwerke, die Ekaterinburger und Kamensker Werke, vier Werke in Perm sowie 124 private Werke unter ihre Verwaltung. Aus diesem Kontext heraus ist es verständlich, dass die Kanzlei vorhatte, auch die fünf Werke von Luginin unter ihre Verwaltung zu bringen. Desto mehr verwundert es, dass es Knauf gelang, vier Werke der Staatshand zu entreißen.

Die Ereignisse überschlugen sich. Für Knauf war es ein Wettlauf mit der Zeit. Als die Kanzlei am Ural den Senatserlass erhielt, beschloss man beim Zlatouster Werk eine Bergdirektion mit zwei Mitgliedern, einem Sekretär, Kassenwart und Buchhalter zu schaffen. Nach diesem Projekt sollten der Bergdirektion alle Werke im Orenburger Oberbezirk, dem auch der Zlatouster Unterbezirk angehörte, unterstellt werden. Das Bergkollegium unterstützte diese Initiative nicht mehr, da es mit Knauf am 30. September 1800 den Vertrag unterzeichnete. Die Privatunternehmer hatten in manchen Regierungskreisen in St. Petersburg entschiedene Fürsprecher, bei denen sich auch Knauf Gehör verschaffen konnte [Тулисов, 1998, с. 37-40].

Andrej Knauf kam nach Russland zu einer Zeit, als dort viele Dynastien bedeutender Unternehmer, die im Ural die Schwerindustrie aufgebaut hatten, am Aussterben waren. Die Nachkommen dieser Unternehmer waren Nutznießer ihrer Vermögen und interessierten sich in der Regel wenig für ihre Betriebe oder wollten sie bei der ersten Gelegenheit gar wieder loswerden. Knauf und seine Partner erkannten diesen Zeitgeist und versuchten Gewinn daraus zu schlagen. Der Export vom Roheisen hatte noch wenige Jahre zuvor geboomt. Was sie nicht sofort erkennen konnten: dass die Ausfuhr ihren Höhepunkt bereits überschritten hatte und nie wieder eine solche Bedeutung erreichen sollte. Bereits in den Jahren 1793 bis 1801 ging die Ausfuhr nach England um ein Drittel zurück, um sich bis 1817 nochmals zu halbieren [Amburger, 1982, S. 127]. Angesichts dieser Exportkrise des russischen Eisens ist es verständlich, dass Knauf dem Trend hin zur Erweiterung des Sortiments und zu Inno- 
vationen gerecht werden wollte. Die Lage der Schwerindustrie auf dem Ural konnte er leider nicht so schnell ändern. Die altertümlichen Methoden in der Wirtschaft waren noch Anfang des 20. Jahrhunderts zu spüren.

Бакшаев А. А. Складывание и функционирование горнозаводского хозяйства Гороблагодатского округа Урала в XVIII - пер. пол. XIX века : автореф. дис. ... канд. ист. наук. Екатеринбург, 2006. [Bakshaev A. A. Skladyvanie i funktsionirovanie gornozavodskogo khozyajstva Goroblagodatskogo okruga Urala v XVIII - per. pol. XIX veka : Avtoref. dis. ... kand. ist. nauk. Ekaterinburg, 2006.]

ГАСО. Ф. 24. [GASO. F. 24.]

Гусякова Т. К. Заводское хозяйство Демидовых в первой половине XIX века : автореф. дис. ... канд. ист. наук. М., 1996. [Gusyakova T. K. Zavodskoe khozyajstvo Demidovykh v pervoj polovine XIX veka : avtoref. dis. ... kand. ist. nauk. M., 1996.]

Дашкевич Л. А., Микитюк В. П. Увеличение численности немецкого населения и его роли в экономике и культуре Урала // Немцы на Урале в XVII - начале XX в. : сб. ст. Н. Тагил, 2009. С. 26-51. [Dashkevich L. A., Mikityuk V. P. Uvelichenie chislennosti nemetskogo naseleniya i ego roli v ekonomike i kul'ture Urala // Nemtsy na Urale v XVII nachale XX v. : sb. st. N. Tagil, 2009. S. 26-51.]

Микитюк В. П. Российско-немецкие предприниматели и их участие в экономической жизни // Немцы на Урале в XVII - начале XX в. Н. Тагил, 2009. [Mikityuk V. P. Rossijsko-nemetskie predprinimateli i ikh uchastie v ekonomicheskoj zhizni // Nemtsy na Urale v XVII - nachale XX v. : sb. st. N. Tagil, 2009.]

Неклюдов Е. Г. Купец А. А. Кнауф и его кредиторы: первый опыт иностранного предпринимательства в горнозаводской промышленности Урала // Изв. Урал. гос. унта. 2004. № 31. Вып. 7. С. 83-101. [Neklyudov E. G. Kupets A. A. Knauf i ego kreditory: pervyj opyt inostrannogo predprinimatel'stva v gorno-zavodskoj promyshlennosti Urala // Izv. Ural. gos. un-ta. 2004. N 31. Vyp. 7. S. 83-101.]

Новиков И. А. И. Ф. Фелькнер и управление Златоустовскими заводами на рубеже XVIII-XIX веков // Краевед. портал Челяб. обл. URL: http://www.kraeved74.ru/pages/ article517.html (дата обращения: 30.04.2011). [Novikov I. A. I. F. Fel'kner i upravlenie Zlatoustovskimi zavodami na rubezhe XVIII-XIX vekov // Kraeved. portal Chelyab. Obl. URL: http://www.kraeved74.ru/pages/article517.html (data obrashcheniya: 30.04.2011).]

Покровский М. Русская история с древнейших времен. Т. 4. M., 1911. [Pokrovskij M. Russkaya istoriya s drevnejshikh vremen. T. 4. M., 1911.]

ПС3 1. Т. 5, № 3464; Т. 26, № 19583. [PSZ 1. Т. 5, N 3464; Т. 26, N 19583.]

РГАДА. Ф. 271. [RGADA. F. 271.]

РГИА. Ф. 37; Ф. 560. Оп. 3. Д. 96 (1817): Кнауф, купец. О расчете с ним по Златоустовским заводам; Ф. 1374. оп. 2. д. 1779: Об уплате долгов; д. 1781 (1799): Кнауф, московский купец. О передаче ему в управление бывших Лугининских заводов; Оп. 3, д. 2500 (1800): Об отдаче в потомственное содержание. Правительственный Сенат, канцелярия генерал-прокурора. Переписка по передаче в казну купленных заводов Лугинина московскому купцу А. Кнауфу в потомственную собственность. По прошению Кнауфа о возвращении ему приобретенных заводов; д. 2649 (1800): Кнауф, московский купец. О покупке им медеплавильных заводов. [RGIA. F. 37; F. 560. Op. 3. D. 96 (1817): Knauf, kupets. O raschete s nim po Zlatoustovskim zavodam; F. 1374. op. 2. d. 1779: Ob uplate dolgov; d. 1781 (1799): Knauf, moskovskij kupets. O peredache emu v upravlenie byvshikh Lugininskikh zavodov; op. 3, d. 2500 (1800): Ob otdache v potomstvennoe soderzhanie. Pravitel'stvennyj Senat, kantselyariya general-prokurora. Perepiska po peredache $\mathrm{v}$ kaznu kuplennykh zavodov Luginina moskovskomu kuptsu A. Knaufu v potomstvennuyu sobstvennost'. Po prosheniyu Knaufa o vozvraschenii emu priobretennykh zavodov; d. 2649 (1800): Knauf, moskovskij kupets. O pokupke im medeplavil'nykh zavodov.]

Рожков Н. Русская история в сравнительно-историческом освещении. Т. 7. Л. ; М., 1928. [Rozhkov N. Russkaya istoriya v sravnitel'no-istoricheskom osveschenii. T. 7. L. ; M., 1928.] 
$\begin{array}{cccclr}\text { Тулисов } & \text { Е. Инструкция } & \text { главному } & \text { начальнику } & \text { Екатеринбургского } \\ \text { горного начальства в } 1802 & \text { году // } & \text { Проблемы истории местного }\end{array}$ управления Сибири конца XVI-XX в. : сб. ст. Новосибирск, 1999. C. 33-36. [Tulisov E. Instruktsiya glavnomu nachal'niku Ekaterinburgskogo gornogo nachal'stva v 1802 godu // Problemy istorii mestnogo upravleniya Sibiri kontsa XVI-XX v. : sb. st. Novosibirsk, 1999. S. 33-36.]

Тулисов, E. К 200-летию открытия Канцелярии главного заводов правления «второго бытия» (1797-1802) // Екатеринбург - вчера, сегодня, завтра : сб. ст. Екатеринбург, 1998. Ч. 1. С. 37-40. [Tulisov, E. K 200-letiyu otkrytiya Kantselyarii glavnogo zavodov pravleniya «vtorogo bytiya» (17971802) // Ekaterinburg - vchera, segodnya, zavtra : sb. st. Ekaterinburg, 1998. Ch. 1. S. 37-40.]

ЦИАМ. Ф. 2; 32. [TSIAM. F. 2; 32.]

Amburger Erik. „Knauff, Andreas“// Neue Deutsche Biographie. 1979. 12. S. 161-162. [online]. Available from: http://www.deutsche-biographie.de/pnd136084036.html

Amburger Erik. Andreas Knauff und die Knauffschen Hüttenwerke im Ural. Sammelband: Fremde und Einheimische im Wirtschafts- und Kulturleben des Neuzeitlichen Russlands. Ausgewählte Aufsätze. Hrsg. v. Klaus Zernack. Wiesbaden (Quellen und Studien zur Geschichte des östlichen Europa 17), 1982. S. 122-130. 1964.

Blum J. Lord and Peasant in Russia. From the Ninth to the Nineteenth Century. N. Y.,

Fremdling Rainer. Technologischer Wandel und internationaler Handel im 18. Und 19. Jahrhundert: die Eisenindustrien in Grossbritannien, Belgien, Frankreich und Deutschland. Berlin, 1986.

Johannsen Otto. Geschichte des Eisens. 2. Auflage, Düsseldorf, 1925.

Verein Deutscher Eisenhüttenleute: Gemeinfassliche Darstellung des Eisenhüttenwesens.

17. Auflage. Stahleisen mbH, Düsseldorf 1970/71.

Wallerstein I. The Modern World-System III. The Second Era of Great Expansion of the Capitalist World-Economy, 1730-1840s. San Diego, 1989.

Ганс Петер Андреас Кнауф (1765 - после 1835), в России - Андрей Андреевич Кнауф - необычный феномен русской истории. Его жизненный путь оставляет много вопросов, ответы на которые до сих пор не найдены. Но в одном можно быть совершенно уверенным: Андрей Кнауф является выдающейся личностью в горнодобывающей и чугунолитейной промышленности Урала.

Уроженец столицы герцогства Гольштейнского города Киля и земляк российского императора Петра III, Андрей Кнауф, сын сапожника без связей и капитала, но талантливый автодидакт, прибыв в Петербург 18-летним юношей, добивается головокружительных успехов на купеческом поприще, становится именитым купцом 1-й гильдии московского купечества и заводчиком на Урале. Исследуется аспект его деятельности на Урале в качестве владельца горнозаводских предприятий, модернизированных за счет трансфера немецких технологий, особенно с 1801 по 1811 г. Его опыт интересен в контексте крепостного права и нехватки квалифицированных кадров при существовавшей системе посессионных заводов. Для разработки данной темы целесообразно все же не ограничиваться метапонятиями «самодержавия» и «крепостничества», служащими интерпретационными образцами исторической картины исследуемого периода, но стараться дополнить их микроисторическими зарисовками, используя «частные» факторы и «роль личности в истории». 
Ключевые слова: Россия; Урал; Андрей Кнауф; индустриализация; горнодобывающая промышленность; металлообрабатывающая промышленность; Урал; модернизация; трансфер технологий.

Andreas Keller (Андрей Викторович Келлер), dr.

Russia, Yekaterinburg

Ural Federal University

a.v.keller@urfu.ru 